\title{
The Effect of Completeness Perception in Narrative Advertising: An Abstract
}

\section{Dongwon Choi and Hyejin Bang}

\begin{abstract}
The current study posits that perceived length of the video ad might be incongruent with the actual length of the video ad, and thus, it might be more influential to viewer's reaction. This study will examine the effect of the completeness of the narrative ad on viewer's perception and attitudinal responses to the ad in the context of online video advertising. We expect that viewers would perceive that incomplete ads are always shorter than complete ads, regardless of their relative lengths. Further, we predict that this perception affects viewer's attitude toward the ad contrarily depending on the context of ad presentation. Specifically, people would prefer a complete ad to an incomplete ad when the ad is presented independently, while people would prefer an incomplete ad to a complete ad when the ads are presented contextually in media content. However, the effect of completeness on people's attitude toward the ad will be eliminated when the actual amount of ad time is explicitly provided.
\end{abstract}

D. Choi $(\bowtie) \bullet H$. Bang

University of Georgia, Athens, GA, USA

e-mail: dc47864@uga.edu; adhjbang@uga.edu 